\title{
BOLUS DYNAMIC COMPUTED \\ TOMOGRAPHY IN THE EVALUATION OF PULMONARY SEQUESTRATION
}

\author{
KENNETH A. BUCKWALTER, MD, BARRY H. GROSS, MD, \\ AND RAMIRO J. HERNANDEZ, MD
}

Three cases of pulmonary sequestration evaluated by bolus dynamic computed tomography are described. The computed tomography appearance and the differential diagnosis are discussed. It is important to recognize the advantages of the bolus dynamic technique in the evaluation of pulmonary sequestration as it may eliminate the need for further studies.

\section{KEY WORDS:}

Lung, abnormalities; Lung, computed tomography;

Computed tomography, contrast enhancement

Pulmonary sequestration may present as an incidental mass noted during computed tomography (CT) of the chest or upper abdomen; conversely, CT may be obtained to evaluate a mass that later proves to be a sequestration. Previous reports of the CT appearance of sequestration have emphasized nonspecific morphologic features (1-3). Appreciation of the vascular nature of this abnormality during dynamic CT following a bolus injection of intravenous contrast may suggest the diagnosis of sequestration. In some patients, CT may even demonstrate the systemic arterial supply of the mass, perhaps obviating the need for angiography. We present three illustra-

From the Department of Radiology, University of Michigan Medical Center, Ann Arbor, Michigan.

Address reprint requests to: Barry H. Gross, MD, Department of Radiology, Box 0030, University of Michigan Medical Center, University Hospital B1D502/0030, 1500 E. Medical Center Drive, Ann Arbor, Michigan 48109-0030.

Received January 1987.

(C) 1987 by Elsevier Science Publishing Co., Inc.

52 Vanderbilt Avenue, New York, NY 10017

$0149-936 \mathrm{X} / 87 / \$ 3.50$ tive cases of pulmonary sequestration diagnosed by bolus dynamic CT.

\section{CASE 1}

A 2-day-old male with tachypnea had a chest radiograph that demonstrated a large, well-circumscribed, noncalcified, homogeneous mass located caudally in the left posterior hemithorax (Figures $1 A$ and $1 B$ ). The differential diagnosis included Bochdalek hernia, neuroblastoma, ectopic kidney, and pulmonary sequestration. Ultrasound examination of the abdomen demonstrated normally situated abdominal viscera. A CT scan of the chest performed the following day showed a large homogeneous mass in the left posterior costophrenic sulcus. Intravenous bolus of contrast mediun demonslrated small vessels in the mass with a large feeding vessel originating from the descending thoracic aorta (Figure $1 C$ ). This appearance was consistent with the suspected diagnosis of pulmonary sequestration, and the patient subsequently went to surgery where a 4-cm solid mass was found in the left lower lobe. The mass had its own pleural covering and had no connection with the bronchial tree. There was a relatively large feeding systemic artery, and a large vein drained the mass at its inferior aspect. Pathologic examination showed numerous dilated air spaces lined by ciliated columnar epithelium without cartilage, confirming the diagnosis of pulmonary sequestration.

\section{CASE 2}

A 37-year-old female with no prior history of pulmonary disease initially presented with pneumonia. She was found to have a hazy opacity in the left lower lobe that failed to clear. The patient was re- 


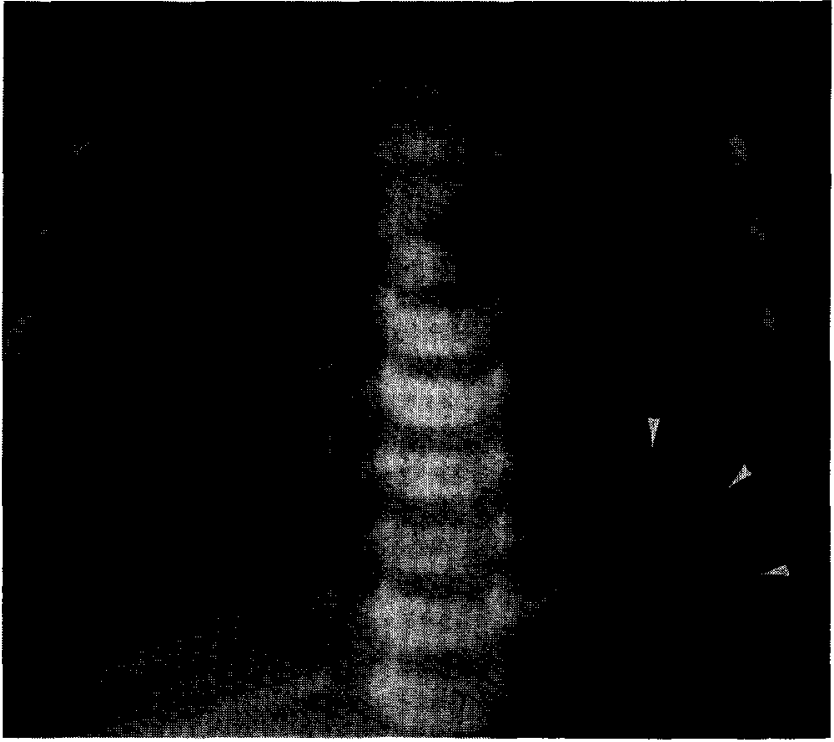

A

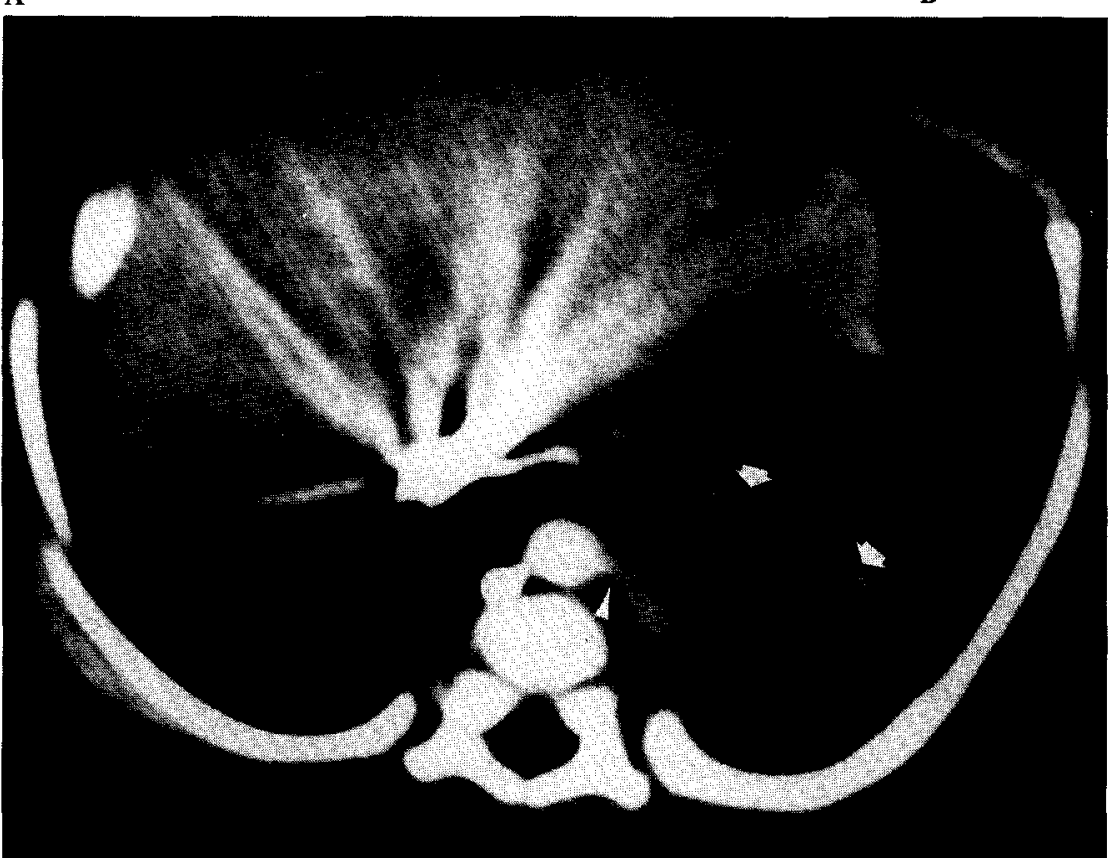

C

ferred to another hospital where a chest radiograph showed a 9-cm rounded mass located posteriorly at the left lung base (Figures $2 A$ and $2 B$ ). A subsequent CT of the thorax showed a heterogeneous mass containing multiple cystic areas in the same location. Intravenous bolus of contrast medium demonstrated branching structures within the mass that enhanced at the same time as the descending aorta (Figure $2 C$ ). The diagnosis of pulmonary sequestration was subsequently confirmed when a flush aortogram (Figure 2D) demonstrated aberrant vessels originating from the descending aorta, supplying the mass.

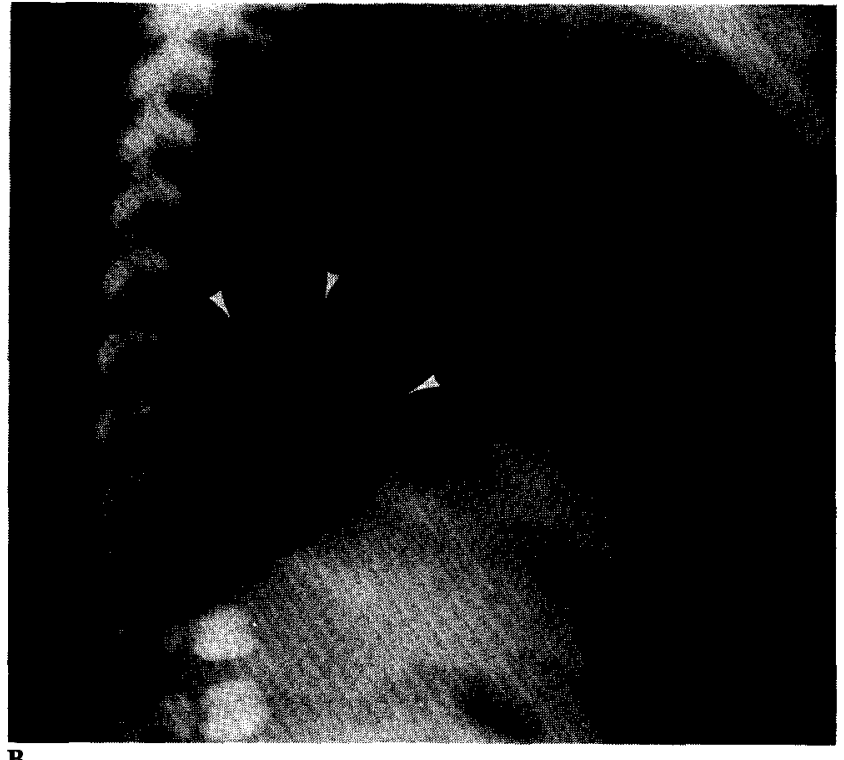

FIGURE 1. (A,B) Patient 1. Anteroposterior and lateral chest radiographs demonstrate a mass (arrowheads) in the left posterior costophrenic sulcus. (C) CT scan following bolus injection of intravenous contrast shows an aberrant systemic vessel (arrowhead) supplying a sequestration (arrows).

\section{CASE 3}

A 44-year-old male with cholangiocarcinoma had a chest radiograph showing dextrocardia and blunting of the right costophrenic angle; the lungs were otherwise normal. Angiography of the liver demonstrated a large aberrant vessel originating from the celiac axis, supplying a small vascular mass located medially at the right lung base (Figure $3 A$ ). Venous drainage was via pulmonary veins, consistent with an intralobar pulmonary sequestration. A subsequent CT scan showed a small, 2-cm oval mass at the medial aspect of the right lung base. Dynamic bolus CT showed intense contrast enhancement 


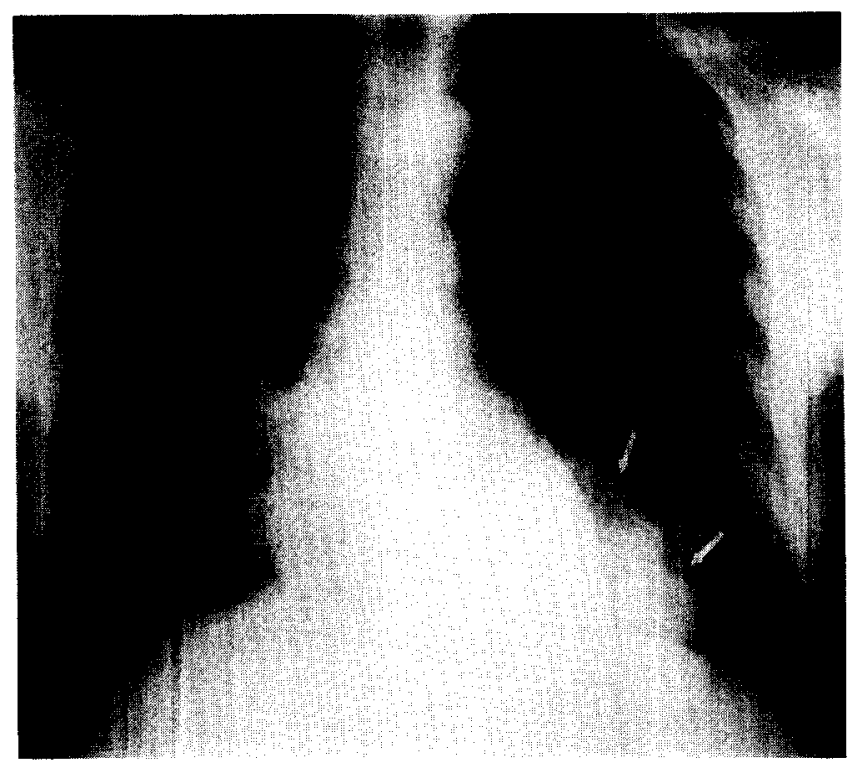

$\mathbf{A}$

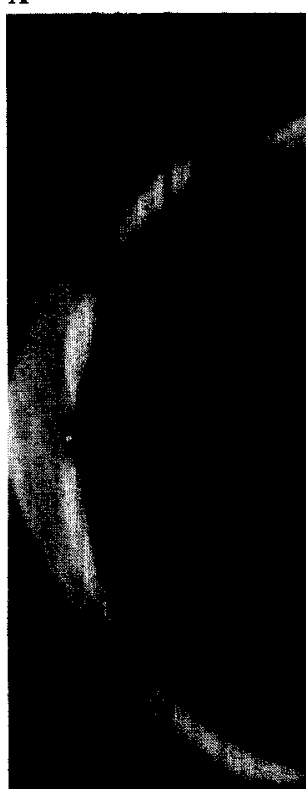

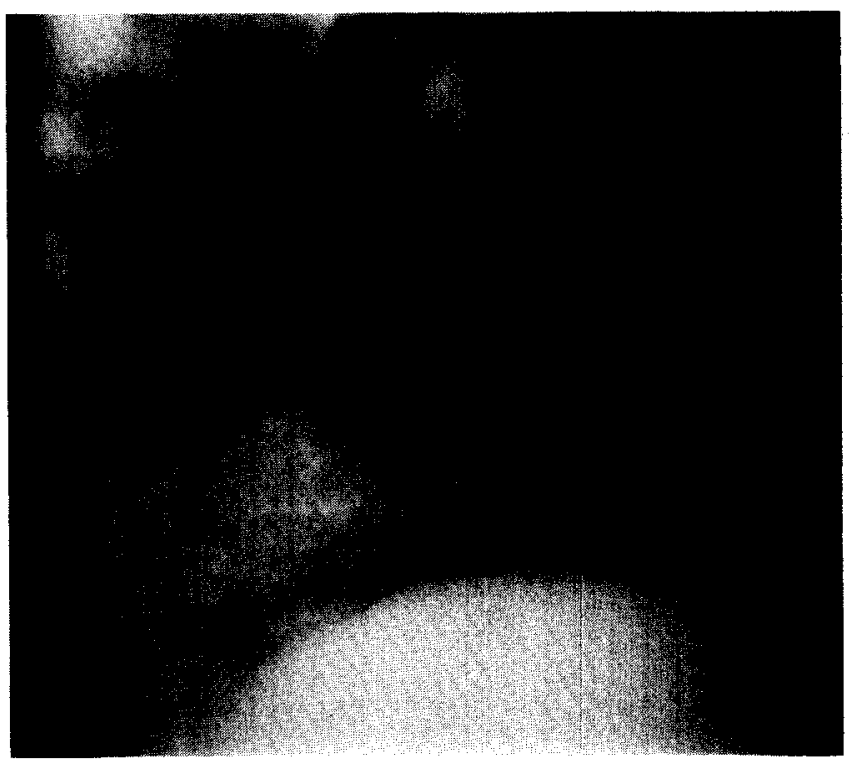

B

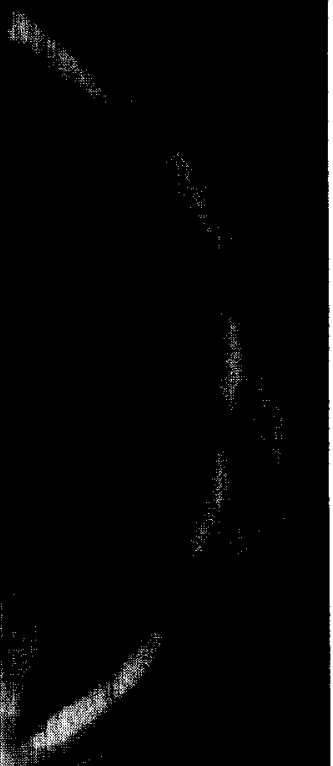

C

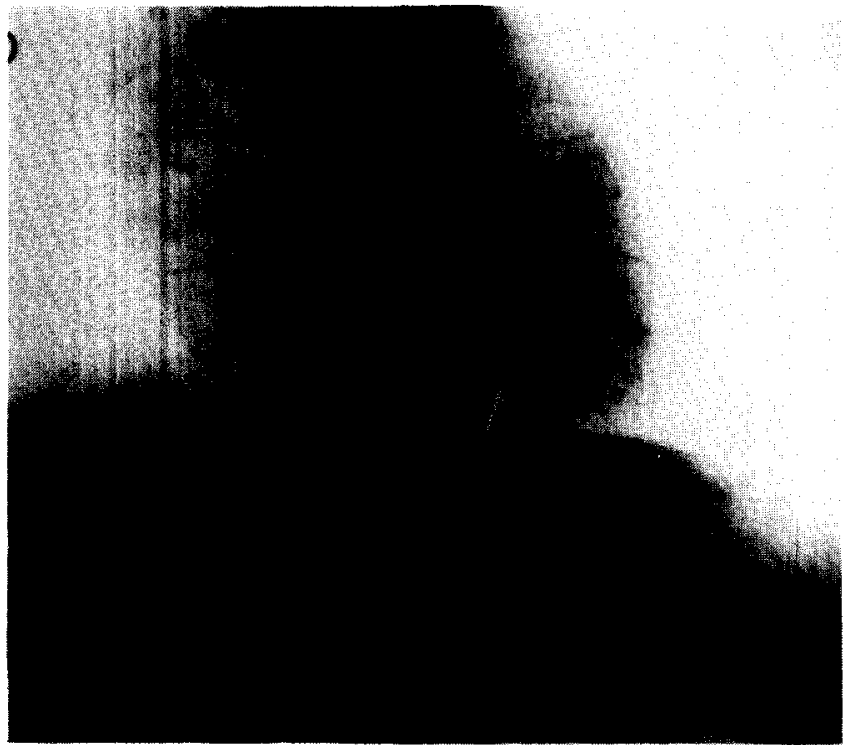

FIGURE 2. (A,B) Patient 2. Posteroanterior and lateral chest radiographs reveal a large mass (arrows) at the left lung base. (C) Bolus CT scan at the level of the mass shows branching structures (arrows) enhancing concurrent with enhancement of the descending aorta. (D) Subtraction view from a flush aortogram confirms that the mass is supplied by a branch from the descending thoracic aorta (arrow). (Courtesy of Dr. Paul Kantzler, Detroit, Michigan). 


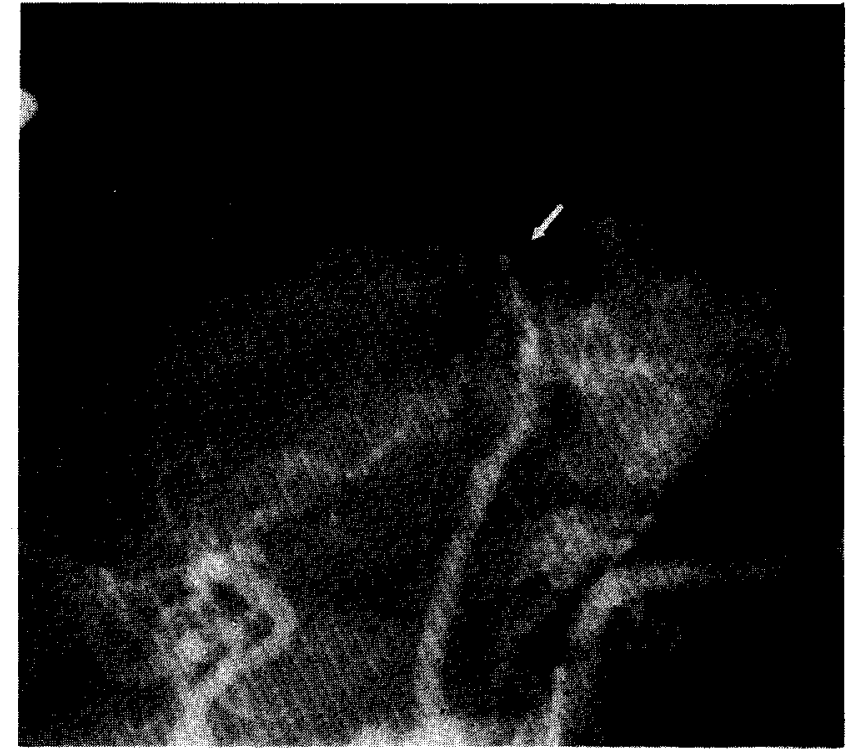

A

FIGURE 3. (A) Patient 3. Celiac axis angiogram, RPO view. A large aherrant vessel (black arrow) supplies a vascular mass at the right lung base (white arrow). Venous drainage was via pulmonary veins. (B) Bolus CT scan also demonstrates the vascular mass (white arrowheads) at the right lung base supplied by the aberrant celiac branch vessel (black arrows).

coincident with the enhancement of the descending aorta (Figure $3 B$ ). The intrahepatic portion of the aberrant feeding vessel was clearly demonstrated.

\section{DISCUSSION}

Pulmonary sequestration is part of the continuum of congenital bronchopulmonary-foregut malformations (4). Sequestrations are usually divided into intralobar and extralobar types (5); both forms are supplied by a systemic artery, frequently a branch of the descending aorta. Intralobar sequestrations are contained within the visceral pleura and generally drain into the pulmonary venous circulation. Extralobar sequestrations have their own pleural investment and drain into the systemic venous circulation via the inferior vena cava, the azygos or hemiazygos system, or the portal venous system. Initially, there is no connection with the bronchial tree in either type of sequestration, and the number of bronchi in the ipsilateral lung is usually normal. Extrapulmonary abnormalities may be present and are more frequently seen with extralobar sequestration. Both forms are most often located inferiorly in the posterior basal segment of a lower lobe, more

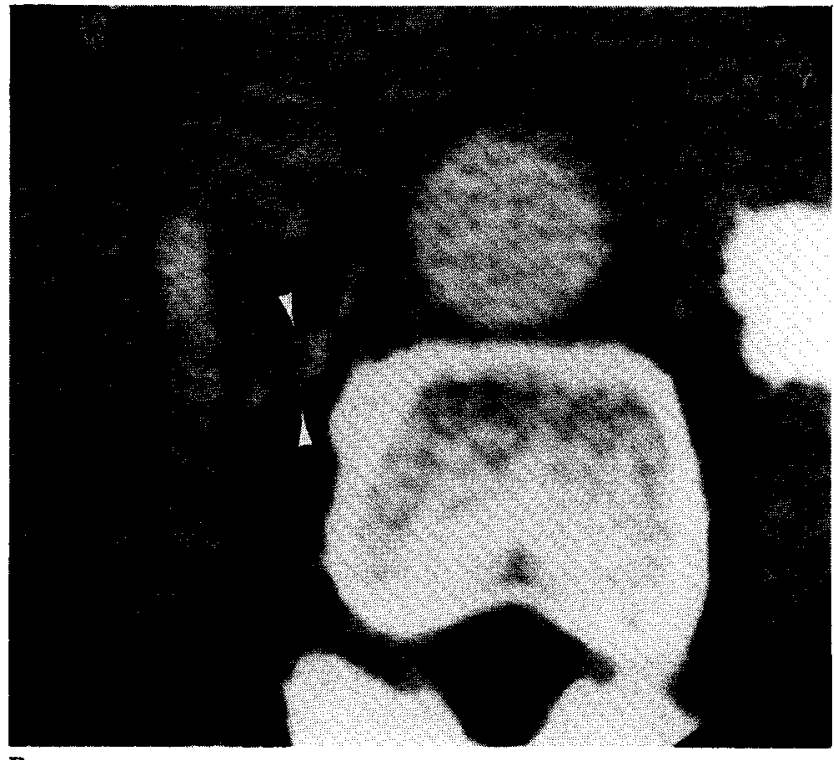

$\mathbf{B}$

commonly in the left than right. Bilateral sequestrations have been reported (6), but they are exceedingly rare.

Both plain film and CT appearances are varied (1-3, 7-9). The mass may be solid or cystic, and it may contain air or air-fluid levels if infected; intralobar sequestration may contain air through collateral air drift. Calcification is rare (10).

Visualization of the systemic arterial vessel supplying the sequestration is necessary for diagnosis. There have been several case reports of demonstration of the feeding artery during contrast-mediumenhanced CT (1-3). The use of bolus dynamic scanning $(11,12)$ facilitated the diagnosis of pulmonary sequestration in the patients we evaluated (Figures 1-3). Even when the feeding artery is not demonstrated, bolus dynamic CT generally demonstrates the vascular nature of a sequestration. CT demonstration of an enhancing vascular lung lesion, especially if located in a lower lobe, should suggest the diagnosis of pulmonary sequestration. Pulmonary parenchymal neoplasms rarely enhance to the same extent (13); zones of low attenuation and demonstration of a feeding artery, features typical of sequestration, are not seen in parenchymal neoplasms.

Collapsed lung may present an appearance similar to sequestration (14), especially if the sequestered lung is infected or communicates with the bronchial tree and contains air. Both entities may present at CT as a heterogeneous mass with areas of high and low attenuation, and both may enhance following infusion of contrast medium. Several 
signs will aid in the distinction of collapse from sequestration. With sequestration, the bronchial anatomy of the ipsilateral lung should be normal and there will be no secondary signs of volume loss. Furthermore, bolus dynamic CT can be extremely useful if the arterial feeder of a sequestration is demonstrated. Time-density plots will provide a more definitive answer if there is still ambiguity. A collapsed lung will show enhancement immediately after the right ventricle, while the enhancement of a sequestration will coincide with that of the descending aorta.

Other vascular lesions, such as pulmonary arteriovenous fistulae and pulmonary vcin varicositics, will enhance following contrast-medium administration (15). It should not be difficult to separate these entities from pulmonary sequestration. Pulmonary arteriovenous fistula generally presents as a round nodule representing a single dilated vessel or a lobulated tangle of serpiginous vessels, usually located in the inner third of the lung. The feeding artery originating in the pulmonary hilum and the draining vein directed toward the left atrium are frequently seen. Time-density plots obtained following a contrast bolus show peak enhancement hetween the enhancement peaks of the right and left sides of the heart. Pulmonary vein varicosities occur less frequently; they are usually asymptomatic and appear as round or oval masses near the left atrium. 'lime-density plots show concomitant enhancement with the left atrium.

Less common vascular lesions, such as arteriovenous malformations with systemic arterial supply (12), chronic pulmonary inflammation with the development of abnormal anastomoses from the systemic to the pulmonary arteries $(16,17)$, and systemic arterial supply to normal basilar segments $(18,19)$, may simulate the CT appearance of sequestration. It may be very difficult to differentiate these uncommon entities from sequestration on the basis of CT. The clinical history may provide inportant clues, and angiography may be required.

\section{REFERENCES}

1. Hochhauser L, Gray RR, St. Louis EL, et al. Computed tomographic diagnosis of pulmonary sequestration. Comput Radiol 1983;8:171-3.

2. Miller PA, Williamson BJR, Minor GR, Buschi AJ. Pulmonary sequestration: visualization of the feeding artery by CT. J Comput Assist Tomogr 1982;6:828-30.

3. Paul DJ, Mueller CF. Case report: pulmonary sequestration. J Comput Assist Tomogr 1982;6:163-5.

4. Gerle RD, Jaretzki A III, Ashley CA, Berne AS. Congenital bronchopulmonary-foregut malformation: pulmonary seque- stration communicating with the gastrointestinal tract. $\mathrm{N}$ Engl J Med 1968;278:1413-9.

5. Fraser RG, Pare JAP. Diagnosis of diseases of the chest. Philadelphia: WB Saunders, 1977:606-12.

6. Wimbish KJ, Agha FP, Brady TM. Bilateral pulmonary sequestration: computed tomographic appearance. Am J Roentgenol 1983;140:689-90.

7. Felson B. The many faces of pulmonary sequestration. Semin Roentgenol 1972;7:3-15.

8. Baker EL, Gore RM, Moss AA. Retroperitoneal pulmonary sequestration: computed tomographic findings. Am J Roentgenol 1982;138:956-7.

9. Thornhill BA, Kyunghee CC, Morehouse HT. Gastric duplication associated with pulmonary sequestration: CT manifestations. Am J Roentgenol 1982;138:1168-71

10. Van Dyke JA, Sagel SS. Calcified pulmonary sequestration: CT demonstration. J Comput Assist Tomogr 1985;9:372-4.

11. Godwin JD, Webb WR. Dynamic computed tomography in the evaluation of vascular lung losions. Radiology 1981;138:629-35.

12. Webb WR. CT of solitary pulmonary vascular lesions. Semin Roentgenol 1984;3:189-98.

13. Halbsguth A, Schulze W, Ungeheuer E, Hoer PW. Pitfall in the CT diagnosis of pulmonary arteriovenous malformation. J Comput Assist Tomogr 1983;7:710-12

14. Glazer HS, Aronberg DJ, Van Dyke JA, Sagel SS. CT manifestations of pulmonary collapse. In: Siegelman SS, ed. Computed tomography of the chest. Philadelphia: Churchill Livingstone, 1984;81-119.

15. Moss AA, Gamsu G, Genant HK. Computed tomography of the body. Philadelphia: WB Saunders, 1983:336-41.

16. Botenga ASJ. The role of bronchopulmonary anastomoses in chronic inflammatory processes of the lung. Am J Roentgenol 104:829-37.

17. Webb WR, Jacobs RP. Transpleural abdominal systemic aTtery-pulmonary artery anastomosis in patients with chronic pulmonary infection. Am J Roentgenol 1977;129:233-6.

18. Kirks DR, Kane PE, Free EA, Taybi H. Systemic arterial supply to normal basilar segments of the left lower lobe. Am J Roentgenol 1976;126:817-21.

19. Flisak ME, Chandrasekar AJ, Marsan RE, Ali MM. Systemic arterialization of the lung without sequestration. Am J Roentgenol 1982:;138:751-3.

\section{CONTINUING MEDICAL EDUCATION QUESTIONS}

1. Which of the following statements does not apply to intralobar pulmonary sequestration?

a. Intralobar sequestrations are contained within the visceral pleura.

b. Initially, there is a direct connection with the bronchial tree.

c. The sequestered segment may contain air through collateral air drift.

d. Venous drainage is usually via the pulmonary venous circulation.

2. The CT appearance of pulmonary sequestration can rarely be simulated by all of the following except:

a. Arteriovenous malformation with systemic arterial supply.

b. Chronic pulmonary inflammation with systemic anastomoses to pulmonary arteries. 
c. Pulmonary vein varicosities.

d. Systemic arterial supply to normal basilar segments.

3. Which of the following statements about pulmonary sequestration are false?

a. Pulmonary sequestration occurs most often in the posterior basal segment of the left lower lobe.

b. Bilateral sequestratons are rare.

c. Calcification occurs frequently.

d. Pulmonary sequestration may contain air if infected.
4. Which of the following statements does not apply to the CT appearance of pulmonary sequestration?

a. Pulmonary parenchymal neoplasms rarely enhance to the same degree as sequestration.

b. Enhancement of a sequestration during bolus dynamic CT coincides with right ventricular enhancement.

c. Bronchial anatomy of the ipsilateral lung is normal.

d. Zones of low attenuation within a basal mass and demonstration of a feeding artery are a typical appearance of pulmonary sequestration. 